\title{
Discussion Notes on "Slip velocity during the flow of a liquid over a solid surface", by E. Ruckenstein
}

\section{J.J. Feng ${ }^{\mathrm{a}}$}

Department of Chemical and Biological Engineering, and Department of Mathematics, University of British Columbia, Vancouver, BC V6T 1Z2, Canada

Received 02 June 2010 / Received in final form 15 June 2011

Published online 30 August 2011

Abstract. Comments are provided for [5].

This article reviews a model that relates the slip velocity to the molecular interaction between solid substrate and the fluid species, especially at the contact line. I find the ideas reasonable and interesting, and will supply additional information that connects these with more recent ideas in the literature.

1. Apparent slip. The attribution of large apparent slip to a gaseous film on hydrophobic surfaces has been supported by recent experiments in microfluidic devices. See, for example, Ref. [1]. More recently, Gao and Feng [2] have done detailed computations of contact line motion and interfacial evolution leading to the gas film.

2. True slip. The idea that a locally peaked force due to gradients of some interaction potential at the contact line gives rise to slip is conceptually consistent with a recent model by Qian et al. [3] based on a generalized Navier boundary condition (GNBC). In this latter model, the slip is related to the "uncompensated Young stress", which arises when the dynamic contact angle $\theta_{d}$ is driven away from the static value $\theta_{s}$ by dynamic flow (at non-equilibrium). However, the algebraic forms differ in the end. The GNBC model relates the slip velocity $v^{\text {slip }}$ to $\gamma\left(\cos \theta_{d}-\cos \theta_{s}\right) \approx \gamma\left(\theta_{s}^{2}-\theta_{d}^{2}\right) / 2$, as opposed to Eq. (29) which has an additional $\theta_{d}$. In addition, the phase-field and MD computations by Qian et al. show a profile of the slip velocity along the wall with a wide $1 / x$ power-law region. This differs from the $1 / x^{4}$ profile given by the current theory. Incidentally, a derivation by Yue and Feng [4] relates the deviation $\cos \theta_{d}-\cos \theta_{s}$ to the capillary number of the flow.

\section{References}

1. J. Ou, B. Perot, J.P. Rothstein, Phys. Fluids 16, 4635 (2004)

2. P. Gao, J.J. Feng, Phys. Fluids 21, 102102 (2009)

3. T. Qian, X.P. Wang, P. Sheng, Commun. Comput. Phys. 1, 1 (2006)

4. P. Yue, J.J. Feng, Phys. Fluids 23, 012106 (2011)

5. E. Ruckenstein, Eur. Phys. J. Special Topics 197, 203 (2011)

\footnotetext{
${ }^{a}$ e-mail: jfeng@chbe.ubc.ca
} 